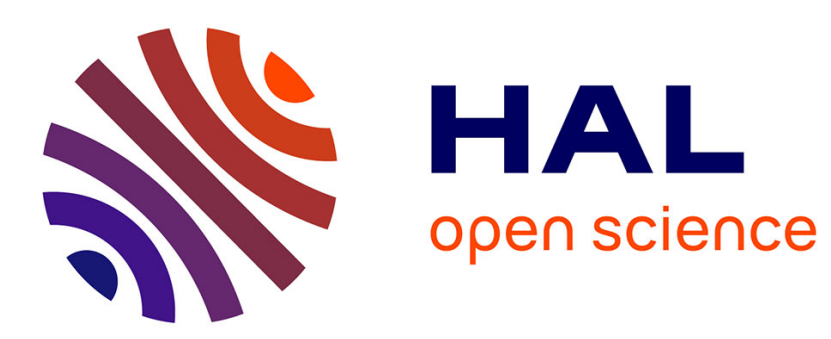

\title{
De la vocation artistique à la précarité: devenir professeur de danse jazz
}

Sébastien Fleuriel

\section{To cite this version:}

Sébastien Fleuriel. De la vocation artistique à la précarité: devenir professeur de danse jazz. Sociologie de l'art, 2010, 15, pp.137-157. 10.3917/soart.015.0137 . halshs-01145228

\section{HAL Id: halshs-01145228 \\ https://shs.hal.science/halshs-01145228}

Submitted on 11 Jun 2017

HAL is a multi-disciplinary open access archive for the deposit and dissemination of scientific research documents, whether they are published or not. The documents may come from teaching and research institutions in France or abroad, or from public or private research centers.
L'archive ouverte pluridisciplinaire HAL, est destinée au dépôt et à la diffusion de documents scientifiques de niveau recherche, publiés ou non, émanant des établissements d'enseignement et de recherche français ou étrangers, des laboratoires publics ou privés. 
Sébastien Fleuriel

Professeur

Université de Lille 1, CNRS, Clersé

Adresse postale :

Institut de sociologie et d'anthropologie

Bâtiment SH2

Université de Lille 1

59655 Villeneuve d'Ascq cedex

De la vocation artistique à la précarité : devenir professeur de danse jazz 


\title{
De la vocation artistique à la précarité : devenir professeur de danse jazz
}

\author{
«Si on est prise nulle part, on peut toujours être prof ${ }^{1}$. »
}

\section{Introduction}

\begin{abstract}
«C'est difficile de combiner à la fois une passion et à la fois être dans le monde réel et être dans un métier, dans le système dans lequel on est et dans la société dans laquelle on est, ça fonctionne comme ça. Donc quand on fait le choix de vivre de ce qu'on aime vraiment c'est..., ça veut dire qu'il va falloir à un moment donné laisser de côté le plaisir de la passion pour se coltiner quelque chose d'un peu plus réel et d'un peu moins réjouissant ${ }^{2}$.»
\end{abstract}

Applicables à l'ensemble des univers artistiques, ces propos sont ici exprimés par une danseuse de jazz professionnelle, Clara, âgée de 24 ans au moment de l'entretien. Ils font référence à une tension caractéristique du champ artistique selon laquelle les aspirations proprement vocationnelles, désignées en l'espèce sous le vocable de passion, entrent en concurrence avec «le monde réel », c'est-à-dire les nécessités de subvenir à ses besoins par l'exercice d'un métier. Pour cette danseuse, la passion renvoie au projet de danser sur scène pour le compte d'une compagnie ou d'une comédie musicale, forme de consécration absolue de la formation professionnelle qu'elle a reçue pendant trois années après le bac dans une école de danse jazz parisienne ${ }^{3}$. Par opposition à ce rêve, le monde réel dont parle Clara correspond au travail alimentaire auquel elle doit s'astreindre chaque jour en assurant le cachet dans des soirées privées par l'intermédiaire d'une agence spécialisée dans l'événementiel et, surtout, en donnant de multiples cours de danse dans différentes écoles de la région parisienne.

La tension entre le projet vocationnel et les nécessités alimentaires n'est pas le produit instantané d'un choix cornélien à effectuer entre deux alternatives : interpréter la danse ou bien l'enseigner. Elle s'inscrit dans l'histoire même de la danse de spécialité jazz ${ }^{4}$ et de son marché du travail toujours embryonnaire en France. Mais elle s'engendre et se perpétue surtout dans les lieux de formation spécialisée des danseurs et danseuses, qui en tant qu'instances de conservation et reproduction de la spécialité, en assurent la survie vis-à-vis de ses concurrentes, les danses classique et contemporaine.

\footnotetext{
${ }^{1}$ Entretien avec une danseuse jazz du 8 mars 2002.

${ }^{2}$ Entretien avec Clara, danseuse de jazz, du 23 mars 2007.

${ }^{3}$ Mauger G. (dir.), L'Accès à la vie d'artiste. Sélection et consécration artistiques, Paris: Les éditions du Croquant, 2006.

${ }^{4}$ Seguin, E., Histoire de la danse jazz. Paris, Chiron, 2003.
} 
En focalisant notre observation sur une des seules écoles de danse de jazz privée bénéficiant d'une reconnaissance du Ministère de la Culture, nous avons cherché à restituer comment celle-ci, forte de sa notoriété, travaille à travers un apprentissage corporel intense ${ }^{5}$, les catégories de perceptions et de compréhension du monde de la danse jazz auprès de sa clientèle. Loin d'être seulement technique, l'incorporation du métier de danseuse ${ }^{6}$ parvient à imposer sa propre doxa selon laquelle toutes les activités alimentaires sont disqualifiées au profit exclusif de la scène, indépendamment de toutes conditions d'existence. Pour ne cependant pas s'en tenir aux effets ponctuels de l'école, l'observation s'est doublée du suivi continu de la trajectoire de Clara en particulier, depuis son entrée en formation jusqu'à trois années après sa sortie, soit pendant plus de 6 ans, avec des entretiens d'étape réguliers.

L'objectif de ce travail à long terme est triple. Il s'agit d'une part de questionner comment s'expriment les aspirations initiales des danseurs et danseuses au moment de l'entrée en formation et comment celles-ci sont travaillées pendant toute la durée de l'apprentissage pour consacrer le métier de danseur interprète comme figure idéalisée de l'artiste danseur. D'autre part, cette doxa n'interdit nullement, à condition qu'ils soient compris comme tels, les multiples aménagements permettant de subvenir aux besoins matériels et financiers des danseuses, dont l'enseignement et le cachet commercial composent la ressource principale. En dépit de cette réalité, qui commande aux danseuses de s'adonner principalement aux activités les moins valorisées du domaine, il s'agit alors de comprendre comment s'effectue la permanence de la croyance et le maintien d'une vocation et d'un projet à danser sur scène constamment suspendu à d'hypothétiques auditions pour des compagnies ou des comédies musicales aussi rares que sélectives.

\section{1 - Les aspirations des danseuses en formation : prédilection à la danse}

Ce n'est pas le fait du hasard si près de 62,5\% des danseuses expriment à leur entrée en premier vœu le souhait de devenir interprètes professionnelles contre seulement 31,9\% aspirant devenir professeures de danse, la proportion s'inversant pour les vœux de second rang, soit $31,8 \%$ interprètes contre $54,5 \%$ professeures (pour la collecte de ces données, cf. encadré 1). Ce choix traduit un ordre de priorité très largement conforté par les dirigeants de

\footnotetext{
${ }^{5}$ Faure, S., Apprendre par corps. Socio-anthropologie des techniques de danse, Paris, La Dispute, 2000.

${ }^{6}$ On ne parlera plus ici que de danseuses dans la mesure où nous n'avons rencontré que deux artistes masculins en formation sur l'ensemble des promotions.
} 
l'école eux-mêmes pour qui la vocation artistique doit d'abord s'exprimer par la scène, bien avant l'enseignement. Ces activités sont en effet pensées dans des registres différents avec les contraintes propres au spectacle d'une part, et celles de l'apprentissage et de la transmission d'un savoir pratique de l'autre. Dans un ordre séquentiel idéal, le métier de professeur peut, et doit, se nourrir de l'expérience préalable de l'interprétation pour transmettre son message. C'est par ces termes que la directrice administrative de l'école accueillait les danseuses qui venaient de satisfaire avec succès à l'audition d'entrée en formation :

\begin{abstract}
« Il faut qu'on vous dise aussi qu'on souhaite former des danseurs interprètes avant de former des professeurs. Parce que notre but est de former des danseurs. Bon c'est bien, le professorat c'est un diplôme, on est d'accord. [...] On n'est pas là pour les freiner, on n'est pas là pour pousser qui que ce soit, à vous de savoir de ce que voulez faire de votre entrée dans notre école : une carrière de danseur interprète avec des possibilités de danser que l'on va vous offrir ou une carrière de professeur, c'est bien aussi, mais c'est très difficile de mener les deux en même temps, il y en quelques uns mais c'est très rare r. $^{7}$
\end{abstract}

Nul ne saurait donc mieux enseigner que celui qui a d'abord expérimenté la scène. La hiérarchie des choix semble d'autant plus signifiante qu'elle fait largement écho aux propres vécus des deux principaux dirigeants de l'école que sont le directeur artistique et la directrice administrative, dont les trajectoires respectives attestent d'expériences scéniques préalables au métier d'enseignant. Le premier, né à Houston en 1958, jouit en effet d'une réputation internationale après avoir côtoyé dans les années 1970 une prestigieuse compagnie de danse jazz new-yorkaise, la Alvin Ailey American Dance Theater, avant d'émigrer définitivement en France en 1979. Son origine américaine compose une réelle ressource symbolique qui contribue alors à asseoir la légitimité du danseur et le constitue en passeur ou ambassadeur de la danse jazz en France à un moment où celle-ci n'est pas encore très diffusée ${ }^{8}$. La seconde $\mathrm{a}$, pour sa part, principalement dansé à Paris au sein de compagnies de danse espagnole à la même période avant de devenir professeur d'éducation physique et sportive et puis professeur de danse dans une université de la région parisienne ${ }^{9}$.

\title{
Encadré 1 : ethnographie de l'école de danse jazz professionnelle
}

Dirigée conjointement par un directeur artistique renommé, et par sa directrice administrative, l'école concentre sur la région parisienne une grande partie des ressources propres à la sauvegarde et à la promotion de la danse jazz en France : formation au diplôme d'Etat de professeur de danse, centre d'examens pour certaines épreuves du diplôme, reconnaissance

\footnotetext{
${ }^{7}$ Discours prononcé devant les danseuses reçues à l'audition du $1^{\text {er juin } 2002 .}$

8 «Et on devait venir passer un mois à Paris, et ça fait 23 ans que je suis toujours là. Et c'est exactement comme ça que ça s'est passé, j'ai rien sollicité et tout ce qui m'est arrivé ici c'était pareil. On est toujours venu me solliciter et j'ai jamais rien cherché et 23 ans plus tard je suis toujours là. Et toute ma vie, c'était comme ça, c'est des occasions qui m'étaient venues, c'était ce qui était prédestiné en fait. Alors j'ai pas beaucoup de mérite, je ne sais pas quoi dire, j'ai été toujours au bon endroit au bon moment. » Entretiens des 6 mai et 17 décembre 2002.

${ }^{9}$ Entretien du 18 octobre 2002.
} 
ministérielle permettant d'attribuer le statut étudiant aux élèves, production d'ouvrage ou d'articles sur l'histoire du jazz ${ }^{10}$, hébergement de compagnies de danse jazz, recrutement d'enseignants renommés, échanges internationaux...

Appelé à y enseigner la sociologie du corps depuis 2001, j'ai pu progressivement acquérir une position privilégiée pour assurer la collecte systématique de matériaux ethnographiques de nature très variée. Ainsi, la distribution d'un court questionnaire auprès d'une promotion de danseuses $(n=72)$ a permis de situer socialement les élèves. Ce travail est venu compléter une série d'observations régulières effectuées aux différents moments forts de la vie de l'école tels que l'audition d'entrée, les spectacles de gala ou de fin d'années, etc. Il a également été ponctué de deux entretiens approfondis avec le directeur artistique, d'un entretien semi-directif avec la directrice administrative, ainsi qu'une série d'entretiens ethnographiques ${ }^{11}$ avec des danseuses des différentes promotions $(n=11)$. Enfin, le travail s'est enrichi du suivi longitudinal de Clara pendant six ans avec un entretien annuel visant à dresser le bilan de l'année écoulée. Un premier compte-rendu du fonctionnement de l'école et de son travail de socialisation a été livré dans Fleuriel, S., «La Danse : entre sociologie de l'art et sociologie du sport », dans Sociologie des arts et de la culture, (sous la direction de S. Girel), Paris, L'Harmattan, 2006, p. 301-315.

Les processus d'incorporation et d'appropriation du métier de danseur ${ }^{12}$ impliquent un investissement ainsi qu'une socialisation exclusivement orientés autour de la danse et produisent une norme de vie propre à l'école. Fonctionnant comme « un monde en soi » ${ }^{13}$, le régime de vie assure relativement l'homogénéité des aspirations professionnelles portées par les danseuses. Dans ce contexte, les parcours des dirigeants s'imposent comme des modèles de professionnalisation réussie dont il convient de suivre l'exemple. Ils conduisent à faire de l'enseignement une sorte de pis-aller, ou dans le meilleur des cas, une assurance contre l'échec éventuel du projet artistique initial : « je voudrais être sur scène avant tout. Mais au cas où, je préfère avoir un diplôme (le diplôme d'Etat de professeur de danse), une sécurité ${ }^{14} \gg$.

Cette hiérarchie des priorités n'est cependant pas le seul produit de l'institution, elle est d'autant mieux intériorisée par les danseuses que celles-ci conçoivent précocement leur activité comme un acte libératoire de toutes les contraintes et pesanteurs sociales. Largement sur-représentées dans les fractions aisées et moyennes de la population (tableau 1), les danseuses envisagent en effet leur pratique comme une forme d'expression pure, délivrée de ses contingences ordinaires : «Je me sens comme dans une bulle, un univers à part, j'oublie qui je suis, je me laisse aller à un jeu où mon corps bouge sans savoir pourquoi et j'y prends

\footnotetext{
${ }^{10}$ Notamment Seguin, E., op. cit. Par ailleurs, l'auteure a collaboré au volumineux Dictionnaire de la danse (sous la direction de Le Moal, P., Paris, Larousse-Bordas, 1999) en y signant de nombreux articles relatifs à l'histoire de la danse jazz.

${ }^{11}$ Beaud, S., «L'usage de l'entretien en sciences sociales. Plaidoyer pour l'entretien ethnographique », Politix, 35, 1996, p. 226-257.

${ }^{12}$ Faure, S., «Les processus d'incorporation et d'appropriation du métier de danseur. Sociologie des modes d'apprentissage de la danse "classique" et de la danse "contemporaine" », thèse de doctorat, Université de Lyon II, Lyon. 1998.

${ }^{13}$ Fleuriel, S., «La Danse : entre sociologie de l'art et sociologie du sport », dans Sociologie des arts et de la culture, (sous la direction de S. Girel), Paris, L'Harmattan, 2006, p. 301-315.

${ }^{14}$ Entretien avec une danseuse du 13 mars 2006.
} 
plaisir. Pour moi, la danse est moyen de m'évader, de ne pas être contrainte, d'être libre ${ }^{15}{ }$. La rhétorique de l'oubli, de l'évasion ou encore la liberté est ainsi un thème fréquemment abordé pour expliquer l'investissement durable dans la pratique. Initiées dès l'enfance à la danse (à l'âge moyen de 6 ans), les danseuses de l'école affichent en moyenne entre treize et quatorze ans de pratique, et ne découvrent en réalité les questions de professionnalisation que très tardivement dans leur carrière artistique. Cette remarque justifie en soi que l'activité n'entre pas nécessairement en prise avec la réalité des conditions matérielles d'existence, et encore moins avec celle d'une professionnalisation effective, l'enjeu initial et symbolique n'étant précisément pas là : «Dans un cours, un spectacle ou autre, j’ose faire des choses que jamais je ne ferais dans la "vraie vie"16 ». Danser étant par définition une manière d'échapper au monde, la question du débouché professionnel reste en définitive longtemps accessoire dans l'expression du projet global, celle des moyens de subsistances s'en trouvant du même coup reléguée au registre d'une priorité secondaire au regard du projet artistique.

Tableau 1 : Origine sociale des danseuses inscrites à l'école

\begin{tabular}{|r|r|r|r|r|}
\hline & \multicolumn{2}{|c|}{ PCS du père } & \multicolumn{2}{c|}{ PCS de la mère } \\
\hline & Effectifs & \multicolumn{1}{c|}{$\%$} & \multicolumn{1}{c|}{ Effectifs } & \multicolumn{1}{c|}{$\%$} \\
\hline Non réponse & 3 & $4,2 \%$ & - & - \\
\hline Artisans, commerçants, chefs d'ese & 12 & $16,7 \%$ & 10 & $13,9 \%$ \\
\hline Cadres, professions sup. & 27 & $37,5 \%$ & 14 & $19,4 \%$ \\
\hline Professions intermédiaires & 14 & $19,4 \%$ & 18 & $25,0 \%$ \\
\hline Employés & 2 & $2,8 \%$ & 20 & $27,8 \%$ \\
\hline Ouvriers & 11 & $15,3 \%$ & 1 & $1,4 \%$ \\
\hline Retraités & 2 & $2,8 \%$ & 1 & $1,4 \%$ \\
\hline Sans emploi, chômage & 1 & $1,4 \%$ & 8 & $11,1 \%$ \\
\hline Total & 72 & $100,0 \%$ & 72 & $100,0 \%$ \\
\hline
\end{tabular}

\section{2 - Le cercle vicieux des impératifs alimentaires}

En dépit de la puissante dénégation des conditions matérielles d'existence en vigueur dans le microcosme de l'école, les danseuses sont néanmoins contraintes de se soumettre à un principe de réalité selon lequel elles doivent à terme envisager leur professionnalisation. S'agissant d'une école privée payante, les parents sont sérieusement mis à contribution pour financer la formation de leur fille, surtout quand celle-ci vient de province et doit assumer en même temps des frais de logement (dans $51,4 \%$ des cas $)^{17}$. Cette première expérience

\footnotetext{
${ }^{15}$ Danseuse, 22 ans, parents commerçants, 17 ans de pratique. Réponse à la question ouverte « Pourquoi dansezvous? »

${ }^{16}$ Danseuse, 18 ans, père cadre d'entreprise, mère profession intermédiaire, 12 ans de pratique. Réponse à la question ouverte « Pourquoi dansez-vous? »

${ }^{17}$ Le coût annuel d'entrée en Formation Supérieure du Danseur, qui s'étale sur deux ans, plus une année optionnelle, était au minimum de 20000 francs au tournant des années 2000. Il est aujourd'hui, en 2009, de 3885 euros. Ce coût, auquel il faut souvent ajouter un loyer (celui du studio de Clara dans le $12^{\text {ème }}$ s'élève par exemple
} 
d'émancipation familiale s'accompagne alors d'une conquête progressive de l'autonomie qui amène les danseuses à faire leurs premiers pas dans l'emploi artistique pour se constituer un revenu complémentaire (cf. encadré 2). Cours de danse occasionnels, exhibitions au cachet deviennent monnaie courante à un stade avancé de la formation pour dégager un salaire de subsistance. De manière graduelle, les danseuses sont amenées à revisiter sinon l'ordre de leurs priorités, du moins leur regard sur l'état du marché du travail artistique. Celui-ci n'offre quasiment aucun débouché professionnel pour les danseuses interprètes. La danse jazz subit en effet la domination des danses contemporaine et classique qui concentrent l'essentiel des subventions aux compagnies, et occupent du même coup la majeure partie des scènes et des programmations en danse. Alors qu'une enquête développée par le ministère de la culture constatait en 1996 que près de $66 \%$ des Français de 15 ans et plus interrogés sur leur pratique de la danse déclaraient s'être adonnés à la danse moderne jazz (27\% pour la danse classique, $12 \%$ pour la danse contemporaine $)^{18}$, Pierre-Emmanuel Sorignet soulignait pour sa part que :

« L'espace des danses est au début des années 1980 essentiellement organisé autour du ballet classique, du musichall et de l'émergence de compagnie de danse jazz. Le ministère Lang fait le choix d'investir massivement dans la danse contemporaine tout en conservant les subventions au ballet classique (essentiellement en direction de l'Opéra de Paris), le music-hall fonctionnant par son débouché commercial, la danse jazz étant de fait écartée ${ }^{19}$. »

Sylvia Faure réalise le même constat en soulignant que la danse jazz n'atteint pas $2 \%$ du total des cuvres diffusées quand la danse contemporaine représente à elle seule $71 \%$ des spectacles de danse programmés contre $15 \%$ pour la danse classique. L'auteure rappelle par ailleurs qu'en tout état de cause « le marché de la diffusion de la danse reste modéré par rapport aux autres arts vivants que sont la musique ou le théâtre ${ }^{20}$. En somme, la danse jazz, une première fois dominée dans le champ des productions artistiques, l'est une seconde fois dans le champ chorégraphique en y occupant une place très marginale relativement aux autres formes dansées. L'état particulièrement tendu du marché du travail pour les danseuses de jazz est ainsi constitutif du manque de reconnaissance institutionnelle de la spécialité au plan de sa diffusion et de sa programmation.

à 700 euros mensuels) suppose donc budget moyen estimé à minima à 1000 euros mensuels en tenant des charges diverses. Il incite largement les danseuses à penser leur insertion professionnelle effective afin d'amortir les investissements : «Pour rentrer ici, c'est 20000 francs l'année pour les filles qui ne prennent que des cours techniques, et nous, en FSD, c'est 22000 francs l'année, bon un peu plus parce qu'il y a toujours des trucs qui se passent. Et Hélène et moi, on a la chance que nos parents paient nos études, mais ils ne les paieront pas continuellement non plus, c'est pour ça qu'on essaie d'avoir l'EAT le plus vite possible parce... ce n'est pas donné non plus. » Entretien du 8 mars 2002.

${ }^{18}$ Ces données sont tirées de «La Danse en amateur », dans Développement culturel, bulletin des études et de la prospective, 112, Ministère de la Culture, 1996, p. 3.

${ }^{19}$ Sorignet, P.-E., «Un Processus de recrutement sur le marché du travail artistique : le cas de l'audition en danse contemporaine », Genèses, 57, 2004, p. 64-88.

${ }^{20}$ Faure, S., «Les structures du champ chorégraphique français », Actes de la recherche en sciences sociales, 175,2008 , p. 92 . 


\section{Encadré 2 : conquête progressive de l'autonomie de Clara}

\section{Année 1 (2002-2003) - 20 ans}

Clara, fille de médecin gynécologue (d'une mère au foyer) quitte Grenoble pour Paris. Entrée à l'école. Installation en collocation avec une amie. Vit exclusivement de l'allocation parentale. Retour au domicile parental à toutes les vacances scolaires. Partage la vie à l'école avec 3 amies.

Année 2 (2003-2004) - 21 ans

Le groupe d'amies se disperse (un abandon). Passe une partie des vacances chez sa seule amie de l'école. Déménagement de ses parents pour Agen. Envisage de passer l'EAT ${ }^{21}$ permettant de suivre la formation de professeur de danse. Ne rentre plus à toutes les vacances scolaires. Ses parents continuent de subvenir à ses besoins.

Année 3 (2004-2005) - 22 ans

Donne ses premiers cours de danse en colonie de vacances. Echoue aux rattrapages de l'EAT en octobre. Prend des cours de danse dans une autre école. Remplace ponctuellement des professeurs de danse. Fait du babysitting deux fois par semaine. Son amie danseuse abandonne la formation. Rencontre amoureuse avec un infographiste en formation. Ses parents continuent de subvenir à ses besoins.

Année 4 (2005-2006) - 23 ans

Obtient l'EAT en avril. Danse bénévolement dans une compagnie (tournée en Pologne). Continue le babysitting. Suit la formation du diplôme d'état. Continue d'assurer des remplacements comme professeur de danse. Assure quelques dates ponctuelles dans l'événementiel. Toujours en colocation avec son amie de Grenoble. Installation de ses parents à Valence qui continuent de subvenir à ses besoins.

\section{Année 5 (2006-2007) - 24 ans}

Obtient le DE en juin. Donne 8 à 10 heures de cours par semaine dans trois structures différentes. Assure régulièrement des cachets pour une agence événementielle. Parvient à dégager un revenu cumulé de 600 euros mensuels en moyenne. Départ de sa colocataire. S'installe avec son petit ami dans le même appartement qui contribue au loyer. Les parents de Clara continuent de payer la part de loyer restante.

\section{Année 6 (2007-2008) - 25 ans}

Expérience professionnelle en compagnie payée au cachet (deux dates ; à Paris et en Pologne). Réussit à doubler le volume horaire de cours (de 10 heures à 18 heures). Continue de danser pour une agence événementielle. Parvient à dégager 1200 euros nets par mois et devient intégralement indépendante au plan financier.

Paradoxalement, la danse jazz reste la plus enseignée dans les écoles de tous niveaux et connaît un succès sans précédent auprès de ceux qui viennent s'y initier en masse. Ce contexte particulier entretient un déséquilibre de l'offre de travail où il s'avère bien plus aisé d'enseigner que de danser alors que la croyance artistique commande les aspirations contraires. Vécue régulièrement comme une situation provisoire, la professionnalisation semble alors se réaliser à marche forcée au détriment du projet artistique et selon un dispositif «bricolé » à l'exemple de Clara :

«Si je donne quinze ou vingt heures de cours par semaine, ça ira mieux. Avec des heures un peu mieux payées que ce que je suis payée aujourd'hui. Je pense que je peux en trouver des heures à vingt euros, c'est faisable, mais bon ce n'est pas énorme non plus, donc après, c'est aussi de trouver des stages à donner pendant les vacances scolaires, et puis bon, moi, j'ai toujours un contact avec une fille qui me fait travailler pour des bar-mitsvas, des mariages, etc.

\footnotetext{
${ }^{21}$ L'Examen d'Aptitude Technique est une épreuve pratique où sont évaluées les capacités en danse par jury composé de professionnels du milieu. L'EAT conditionne la possibilité de s'inscrire au diplôme d'Etat de professeur de danse.
} 
Donc j'ai à peu près une date tous les mois, c'est cent vingt euros, donc c'est toujours un petit plus dans le mois, mais bon ce n'est pas déclaré, c'est au black, etc. Donc c'est vrai que c'est le système D pour l'instant ${ }^{22}$. »

Le «système D» évoqué par Clara suppose de consacrer beaucoup d'énergie à la reproduction et l'entretien des ressources qui lui permettent son indépendance. Mais il a aussi pour corolaire d'hypothéquer à chaque instant la pérennité du projet artistique en lui confisquant une partie du temps qui lui serait nécessaire, dans une sorte de «dilemme pratique qui découle de l'incompatibilité structurelle entre l'impératif de subvenir à [ses] besoins financiers [...], et la quête de reconnaissance ${ }^{23}$ ». C'est ainsi qu'en 2007, au moment même où Clara accédait à sa pleine autonomie, elle déclinait une audition pour une comédie musicale au motif qu'elle «n'a pas de temps à perdre avec un projet » qu'elle devinait par avance compromis. Comme dans un cercle vicieux, ce sont alors les impératifs alimentaires censés préparer à la vie d'artiste qui en deviennent le principal frein.

\section{3 - Permanence de la croyance et maintien du projet artistique}

Quand bien même le projet artistique reste à l'état d'une simple hypothèse, y renoncer définitivement coûterait cependant trop cher tant il soutient tout l'édifice mis en œuvre. Abandonner toute velléité à devenir danseur interprète reviendrait en effet à rendre injustifiées les multiples situations de petits boulots cumulées pour subvenir aux besoins élémentaires. C'est donc la tension entre les aspirations purement artistiques et les contingences alimentaires qui favorise un équilibre fragile entre toutes les activités. Si l'état du marché du travail oblige à un certain réalisme et force à l'adaptation, le métier de danseuse interprète reste un rêve obligé qui donne du sens à l'ensemble. Deux forces contradictoires travaillent ainsi perpétuellement les danseuses et déterminent le jeu des identités.

D'une part, les premiers pas dans l'emploi bornent drastiquement les aspirations purement artistiques et incitent les danseuses à nuancer leur position sur leurs activités alimentaires. Le cas de Clara (encadré 3) est ici exemplaire de la manière dont elle est amenée à revisiter sa propre condition. D’un rejet radical de l'enseignement la première année («c'est un peu dommage de s'enterrer dans un diplôme d'Etat »), Clara se découvre au terme de sa formation en troisième année une véritable vocation pour l'enseignement («j'ai eu une révélation »). Le déplacement de point de vue s'achève très symboliquement par une véritable transaction identitaire qui lui fait troquer son statut de danseuse professionnelle pour celui de professeure

\footnotetext{
${ }^{22}$ Entretien avec Clara du 23 mars 2007.

${ }^{23}$ Schotté, M., «La condition athlétique. Ethnographie du quotidien de coureurs professionnels immigrés », Genèses, 71, 2008, p. 88.
} 
de danse dans toutes les situations où elle doit se présenter succinctement (« je préfère dire professeur de danse, au moins, [les gens] savent qu'il y a un métier derrière et quelque chose de concret »). Nécessité faisant vertu, Clara tire alors son parti d'une situation qu'elle a d'abord fuie en assouplissant son jugement initial : «J'arrive quand même maintenant à prendre du plaisir et à ne pas me fermer en me disant c'est alimentaire, ça va être nul ».

D'autre part, la doxa du microcosme oblige à tenir le cap en maintenant un projet artistique et en continuant de faire croire au rêve que les danseuses se formulent. Clara persiste encore quelquefois à se présenter à des auditions pour des projets à forte plus-value artistique et parvient sporadiquement à danser sur scène, le plus souvent dans des conditions bien peu satisfaisantes au plan professionnel et financier. En 2007, elle a par exemple pu se produire pour deux dates au sein d'une compagnie provinciale intégralement subventionnée par un Conseil Général. Cette expérience, vécue comme une forme de consécration du milieu («c'était déjà une preuve de reconnaissance, on va dire, [...] c'était vraiment la grosse satisfaction de cette année »), débouche sur la participation à un festival, sous condition de prestations gratuites pour les danseurs néanmoins. Comme le remarque Clara, la croyance est alors le seul moteur qui peut venir justifier un tel investissement :

«Il faut, c'est le propre de l'humain, tu as besoin d'avoir de l'espoir pour avancer. Sans espoir, on ne fait rien dans la vie. Si on ne croit pas que ça peut être réalisable, si on ne croit pas les choses possibles, on ne le fait pas. Donc non, heureusement quelque part. Et en même temps, ce n'est pas amer, parce que je n'ai pas enfoui ce rêve là, je sais qu'il n'est pas réalisable à $100 \%$, je sais que je ne vais pas vivre de la danse en ne faisant que ce que j'aime ${ }^{24}$.»

Plutôt que de les opposer, il faut tenir ensemble les deux facettes de la vie d'artiste pour en saisir la dynamique et apercevoir que l'une et l'autre ne renvoient pas à deux orientations radicalement concurrentes mais plutôt à deux alternatives à la fois inconciliables et cependant complémentaires. Car c'est précisément dans l'effort pour rendre le tout cohérent que se joue l'acceptation de situations de travail artistique hautement improbables quand elles sont prises pour elles-mêmes.

\section{Encadré 3 : Evolution du projet artistique et transaction identitaire de Clara}

Année 1 (2002-2003) - 20 ans

«Moi personnellement, c'est sûr, c'est plus l'aspect danse que professorat. Dès le départ, je savais que je ne serais pas... que je n'étais pas là pour avoir un diplôme d'Etat. Il y a beaucoup de filles qui n'ont pas du tout la motivation pour le faire mais qui veulent quand même le passer le diplôme pour avoir un bagage, un diplôme etc. Je comprends qu'on... pour ne pas prendre de risque, on sait très bien que c'est un milieu où il n'y a pas beaucoup de débouchés, que c'est très dur, que le jour où on se casse une jambe, c'est bien d'avoir prévu la suite, c'est une sécurité d'avoir le diplôme, mais je pense que s'il n'y a pas une vraie motivation, une vraie envie, c'est un peu dommage d'aller s'enterrer dans un diplôme d'Etat. »

\footnotetext{
${ }^{24}$ Entretien du 23 mars 2007.
} 
Année 2 (2003-2004) - 21 ans

«Et finalement pour les danseurs de jazz, on se dit "mais alors nous, quelle est notre place là-dedans ?" Le jazz, ce n'est pas que du divertissement, les paillettes, on va amuser les gens, non. Mais ce n'est pas acquis visiblement parce qu'il n'y a rien, c'est désespérant. C'est désespérant ! Danseuse dans un orchestre, le samedi ? Bon, c'est pas mal, c'est une expérience comme une autre. Mais je veux dire, c'est loin des rêves de petites filles qu'on a quand même [...] je ne veux pas être prof, ce n'est pas mon truc premier, mais disons que je pense que ça peut vraiment m'aider à comprendre certaines choses, le fait d'avoir à expliquer à quelqu'un, ça permet aussi de trouver des choses pour soi-même déjà. »

Année 3 (2004-2005) - 22 ans

« Moi qui étais au départ un peu sceptique par rapport au professorat, ça m'a vraiment... J'ai une révélation en fait pendant cet été, j'ai travaillé avec des enfants, et je me suis éclatée, je me suis découverte moi-même en tant que pédagogue, et ça m'amenait des choses, tout ça quand même m'enrichit, j'ai une autre vision de la danse. Ça m'a redonné confiance en moi, et ça m'a ouvert d'autres perspectives quoi. Il faut que je concrétise, on ne peut pas vivre de la danse uniquement. C'est difficile aujourd'hui, on ne peut pas miser que là-dessus. Donc, pour moi c'est fondamental d'avoir ce diplôme, et je sais que je peux. »

\section{Année 4 (2005-2006) - 23 ans}

« Oui, ça me plaît beaucoup [de donner cours]. Ça me plaît beaucoup, et ça me donne...et ça me donne confiance. Parce que je vois le retour des élèves vis-à-vis de moi et je vois ce que je peux faire. Les années que j'ai vécues ici à Paris, ce que j'ai acquis, pour moi-même, ça c'est de l'acquis pour la vie. Donc en fait, je pense que j'attends, je mets mes pions sur l'échiquier, et j'attends de voir ce qu'il se passe en fonction de ça, et je réajuste. En fait, c'est ça ma technique. Je vais là où ça me semble être juste pour moi, et si je me trompe si la vie me bloque, me ferme des portes, je réajuste. »

\section{Année 5 (2006-2007) - 24 ans}

« Je tourne une page, j'ai fini, j'ai mon diplôme, quoi qu'il arrive maintenant, j'ai quelque chose. Ca valide une compétence. [...] C'est vrai que souvent je dis [que je suis] professeur de danse, et après danseuse. Parce que si je dis danseuse, les gens disent "mais de quoi tu vis? Qu'est-ce que tu fais ? Quand est-ce qu'on peut venir te voir danser ?" Je ne peux pas répondre. Donc je préfère dire professeur de danse, au moins, ils savent qu'il y a un métier derrière et quelque chose de concret. Dans la tête des gens, au moins, il y a une case quelque part, alors que danseur, il n'y a pas réellement de case pour danseur, quoi. »

\section{Année 6 (2007-2008) - 25 ans}

« Il y a eu deux choses différentes, un côté vraiment artistique qui m'intéresse mais où là, il n'y a pas beaucoup de dates, et jouer le jeu du truc alimentaire, et faire des choses qui ne sont pas forcément très élevées d'un point de vue artistique mais avec lesquelles on peut prendre du plaisir quand même et qui me permettent d'avoir des dates quand même. J'arrive quand même maintenant à prendre du plaisir et à pas me fermer en me disant c'est alimentaire, ça va être nul. Parce que sinon... on peut quand même prendre du plaisir tout en sachant que voilà artistiquement ça ne va pas être génial, mais s'amuser quand même avec ça. [...] Donc honnêtement, je trouve que j'ai une qualité de vie, je préfère gagner un peu moins et avoir la vie que j'ai aujourd'hui. [...] Donc en même temps, c'est normal, il faut comprendre les enjeux, et accepter les règles du jeu. »

\section{Conclusion}

La polarisation entre aspirations artistiques et nécessités alimentaires n'est pas une question existentielle pour artistes ratés en mal de création. Elle recèle en elle tous les germes d'une précarité structurelle qui affecte aujourd'hui l'existence d'une partie des artistes ${ }^{25}$. D'un côté, la consécration artistique rime en danse jazz avec une quasi-gratuité rarement remise cause, tant les prétendants en quête de reconnaissance sont nombreux. De l'autre, ces mêmes artistes offrent une armée de réserve peu exigeante pour occuper la myriade d'emplois précaires complaisamment entretenus par les associations parapubliques du secteur culturel, venues

\footnotetext{
${ }^{25}$ Menger, P.-M., Les Intermittents du spectacle. Sociologie d'une exception, Paris, Edition de l'école des hautes études en sciences sociales, 2005.
} 
combler les carences du service public ${ }^{26}$. En 2008, Clara cumule trois emplois de professeur de danse pour trois associations paramunicipales différentes situées dans trois lieux éclatés de la grande couronne parisienne. Payée à la vacation pour deux d'entre elles, au noir pour la dernière, elle doit s'assurer suffisamment de revenus pour s'autofinancer à toutes les vacances scolaires. Adossés l'un à l'autre, ces deux pôles créent donc une redoutable dynamique dans laquelle les artistes s'engouffrent aisément sans parvenir à exercer un quelconque contrôle collectif sur la définition de leur conditions de travail. Ce que faisait remarquer Clara à propos de son emploi non déclaré : «Je ne sais pas, je n'ai rien vu, je ne sais rien. Non, mais moi j'ai besoin de gagner ma vie, elle me paie tous les mois, c'est son problème. Pour moi, c'est cette dame qui est responsable, c'est elle qui a monté son truc, qui fait venir des gens sans déclarer, enfin voilà. C'est sa responsabilité ${ }^{27} \gg$. A ce compte là, les règles du jeu n'ont guère de chance d'évoluer dans un sens positif avant longtemps tant les conditions de reproduction d'un marché précaire semblent profondément installées.

\section{Bibliographie}

Beaud, S., «L'usage de l'entretien en sciences sociales. Plaidoyer pour l'entretien ethnographique », Politix, 35, 1996, p. 226-257.

Faure, S., «Les processus d'incorporation et d'appropriation du métier de danseur. Sociologie des modes d'apprentissage de la danse "classique" et de la danse "contemporaine" », thèse de doctorat, Université de Lyon II, Lyon. 1998.

Faure, S., Apprendre par corps. Socio-anthropologie des techniques de danse, Paris, La Dispute, 2000.

Faure, S., «Les structures du champ chorégraphique français », Actes de la recherche en sciences sociales, 175, 2008, p. 82-97.

Fleuriel, S., «La Danse : entre sociologie de l'art et sociologie du sport », dans Sociologie des arts et de la culture, (sous la direction de S. Girel), Paris, L'Harmattan, 2006, p. 301-315.

Hely, M., «Servir l'intérêt général ou produire de l'utilité sociale ? Avenir de la fonction publique et marché du travail associatif », Les mondes du travail, 5, 2008, p. 23-37.

\footnotetext{
${ }^{26}$ Hely, M., « Servir l'intérêt général ou produire de l'utilité sociale ? Avenir de la fonction publique et marché du travail associatif », Les mondes du travail, 5, 2008, p. 23-37.

${ }^{27}$ Entretien du 28 mars 2008.
} 
Mauger G. (dir.), L'Accès à la vie d'artiste. Sélection et consécration artistiques, Paris: Les éditions du Croquant, 2006.

Menger, P.-M., Les Intermittents du spectacle. Sociologie d'une exception, Paris, Edition de l'école des hautes études en sciences sociales, 2005.

Ministère de la culture, «La Danse en amateur », dans Développement culturel, bulletin des études et de la prospective, 112, Ministère de la Culture, 1996.

Schotté, M., «La condition athlétique. Ethnographie du quotidien de coureurs professionnels immigrés », Genèses, 71, 2008, p. 84-105.

Seguin, E., Histoire de la danse jazz. Paris, Chiron, 2003.

Sorignet, P.-E., «Un Processus de recrutement sur le marché du travail artistique : le cas de l'audition en danse contemporaine », Genèses, 57, 2004, p. 64-88. 